\title{
Quantification of the uncertainties of high-speed camera measurements
}

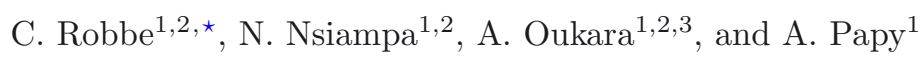

1 Royal Military Academy, Department of Weapons Systems and Ballistics, 30 avenue de la Renaissance, 1000 Brussels, Belgium

2 University of Liège (ULg), Aerospace \& Mechanical Engineering Department (LTAS), 1, Chemin des Chevreuils, 4000 Liège, Belgium

3 Polytechnic Military School of Algiers (EMP), Algeria, 5 Bordj El Bahri, Algiers, Algeria

Received: 25 February 2014 / Accepted: 9 June 2014

\begin{abstract}
This article proposes a combined theoretical and experimental approach to assess and quantify the global uncertainty of a high-speed camera velocity measurement. The study is divided in five sections: firstly, different sources of measurement uncertainties performed by a high-speed camera are identified and quantified. They consist of geometrical uncertainties, pixel discretisation uncertainties or optical uncertainties. Secondly, a global uncertainty factor, taking into account the previously identified sources of uncertainties, is computed. Thirdly, a sensibility study of the camera set-up parameters is performed, allowing the experimenter to optimize these parameters in order to minimize the final uncertainties. Fourthly, the theoretical computed uncertainty is compared with experimental measurements. Good concordance has been found. Finally, the velocity measurement uncertainty study is extended to continuous displacement measurements as a function of time. The purpose of this article is to propose all the mathematical tools necessary to quantify the individual and global uncertainties, to highlight the important aspects of the experimental set-up, and to give recommendations on how to improve a specific set-up in order to minimize the global uncertainty. Taking all these into account, it has been shown that highly dynamic phenomena such as a ballistic phenomenon can be measured using a high-speed camera with a global uncertainty of less than $2 \%$.
\end{abstract}

Keywords: High-speed camera; uncertainties; velocity measurement; displacement measurement; ballistics

\section{Introduction}

During the past fifteen years, the high-speed camera has become an essential measurement tool. Recent technologies can achieve a frame rate up to one million images per second, making it very convenient to assess highly dynamic phenomena such as ballistics. The results consist of measurement of specific displacements as a function of time, or velocity measurements. Thanks to the technological evolution, the high-speed camera is more and more precise, relatively easy to setup, and almost always guarantee no modification of the phenomenon to measure.

However, behind this apparent ease of use and great precision, many optical and geometrical phenomena are implicitly involved when proceeding to an effective measurement. Therefore, measurements are often affected by errors that can be quantified up to a certain range, hence the necessity to use the concept of uncertainty. The measurement uncertainty is defined as the possible error on the value of a quantity to measure, provided

\footnotetext{
^ Correspondence: cyril.robbe@rma.ac.be
}

by the result of the measure. The uncertainty quantifies then the maximum expected error when proceeding to measurements $[1,2]$.

The topic of this article concerns the measurement of the average velocity of a ballistic projectile travelling a specified distance. The presented results remain applicable to any dynamic phenomenon. To clarify the computations, the projectile's movement is supposed to be perfectly straight, always in the same 2D plane, and always horizontal. The employed camera is a Photron SA-5 highspeed camera [3].

The different sections presented in this article are the following:

- determining the useful theoretical concepts in order to proceed to the uncertainty computations;

- identifying and quantifying all the sources of errors and uncertainties involved in the measurement;

- studying their influence on the velocity measurement;

- gathering the results to get the value of the total velocity measurement uncertainty;

- studying the influence of the parameters of the camera set-up on the uncertainty; 


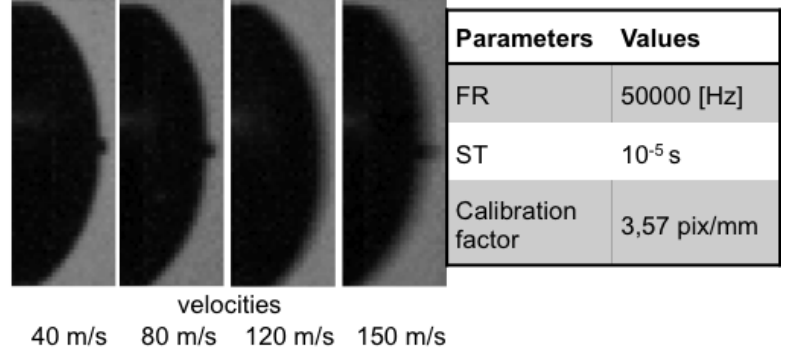

Fig. 1. Illustration of the motion blur when filming a projectile flying at different velocities.

- comparing the results with reference experimental measurements;

- extending the results on the continuous displacement of the projectile measurement as a function of time.

\section{Theoretical background}

\subsection{Definitions}

A few theoretical concepts are necessary to carry out the uncertainty study:

The frame rate $F R[\mathrm{~Hz}]$ is defined as the number of images that a camera can take in one second. A typical camera is characterised by a frame rate of 24 frames per second (FPS), a high-speed camera can go up to 1000000 FPS.

The geometrical resolution res $g$ [pix] is defined as the number of pixels per unit of surface. Many high-speed camera parameters are the result of a compromise between the frame rate and the resolution, meaning that the highest possible frame rates can only be achieved at the price of a reduced geometrical resolution, and vice versa.

The angle of view $\Omega\left[^{\circ}\right]$ describes the angular extent of a given scene that is imaged by the camera.

The shutter time $S T[\mathrm{~s}]$ is the time interval during which the sensor of the camera is exposed at each image capture. The maximal shutter time is equals to the inverse of the frame rate. For filming highly dynamic phenomena such as ballistics, the shutter time is generally reduced. Indeed, when the shutter time is too high in comparison with the velocity of the phenomenon, the movement of the object induces blur around its representation on the image, leading to motion blur [2,4], illustrated in Figure 1.

Mathematically, the motion blur $M B$ [pix] is quantified as shown in equation (1):

$$
M B[\mathrm{pix}]=v_{\text {pix }} S T
$$

where $v_{p i x}[\mathrm{pix} / \mathrm{s}]$ is the velocity of the studied object.

\subsection{The process of a velocity measurement}

The setup to perform a velocity measurement is illustrated on Figure 2. The process to perform the measurement is described as follows.

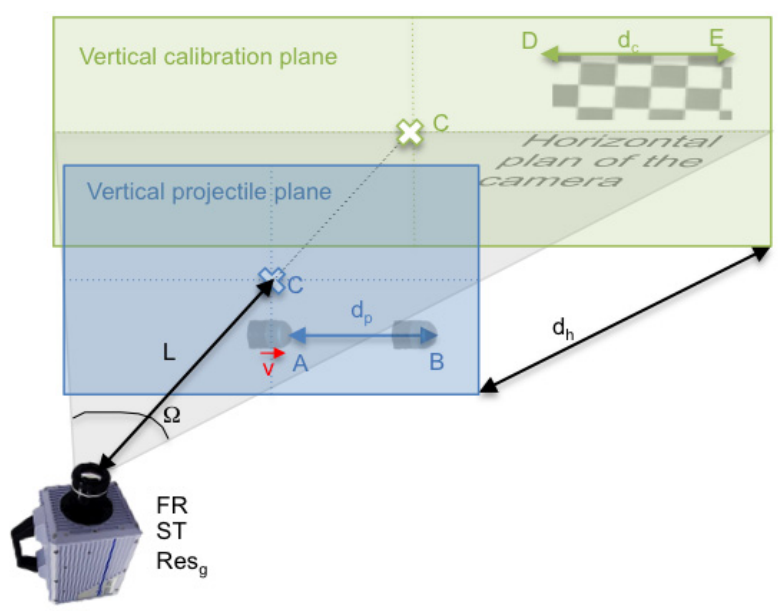

Fig. 2. Illustration of the experimental set-up.

The camera is placed at a certain distance $L[\mathrm{~m}]$ of the projectile's trajectory. The axis of the camera should be orthogonal to this trajectory (as discussed in the section gamma angle uncertainty). A reference object, with one specific dimension $d_{c}[\mathrm{~mm}]$, is placed in a vertical plane. Ideally, the distance $d_{h}[\mathrm{~mm}]$ between this vertical calibration plane and the vertical projectile plane is null (as discussed in the section calibration uncertainties). After taking a picture, the distance $d_{c}$ [pix], which corresponds to number of pixels characterising the distance $d_{c}[\mathrm{~mm}]$, is measured on the image. This measurement allows computing the calibration factor calib [pix/mm], which is the ratio between distances in pixel on the image and real distances. The factor calib can also be determined thanks to the $L$, res $g$ and $\Omega$ parameters:

$$
\operatorname{calib}[\mathrm{pix} / \mathrm{mm}]=\frac{d_{c}[\mathrm{pix}]}{d_{c}[\mathrm{~mm}]}=\frac{\operatorname{res}_{g}}{500 \tan \left(\frac{\Omega}{2}\right) L} .
$$

In the present case, calib is measured thanks to the reference object, which is easier to perform and gives better precision.

Afterwards, the flight motion of the projectile is recorded and $d_{p}$ [pix], corresponding to displacement of the projectile during a certain time interval $\Delta t$, is measured. This measurement is achieved by locating the projectile's position on two different images of the video. The distance $d_{p}[\mathrm{pix}]$ is converted to the real distance $d_{p}[\mathrm{~mm}]$ thanks to the calibration factor calib. The time interval $\Delta t$ is measured by the chronometer of the camera. The velocity $v[\mathrm{~m} / \mathrm{s}]$ can then ultimately be computed (Eq. (3)).

$$
\begin{aligned}
v\left[\frac{\mathrm{m}}{\mathrm{s}}\right] & =\frac{d_{p}[\mathrm{pix}]}{1000 \text { calib }[\mathrm{pix} / \mathrm{mm}] \Delta t[\mathrm{~s}]} \\
& =\frac{d_{p}[\mathrm{pix}] d_{c}[\mathrm{~mm}]}{1000 d_{c}[\mathrm{pix}] \Delta t[\mathrm{~s}]}
\end{aligned}
$$

All the parameters influencing the velocity measurement uncertainty are identified and quantified for the presented set-up in Table 1. 
Table 1. The experimental set-up parameters.

\begin{tabular}{cc}
\hline Parameter & Value \\
\hline$L[\mathrm{~m}]$ & 0.7 \\
$\Omega\left[^{\circ}\right]$ & 22.86 \\
$F R[\mathrm{~Hz}]$ & 50000 \\
$\operatorname{Res}_{g}[\mathrm{pix}]$ & 1024 \\
$S T[\mathrm{~s}]$ & $10^{-5}$ \\
$d_{c}[\mathrm{~mm}]$ & 120 \\
$d_{p}[\mathrm{~mm}]$ & 100 \\
$v[\mathrm{~m} / \mathrm{s}]$ & 100 \\
\hline
\end{tabular}

All these steps are performed thanks to the use of a homemade dedicated tracking software $[2,5]$. An extra objective on the present study is to update this software, taking the presented results into consideration.

\subsection{The uncertainty computation}

The total velocity measurement uncertainty due to different sources of independent linear uncertainties is expressed by equation (4) $[1,4,5]$ :

$$
U_{v}=\sqrt{\sum_{1}^{n}\left(\frac{\partial f}{\partial x_{i}}\right)^{2} u^{2}\left(x_{i}\right)}
$$

where $U_{v}$ is the velocity measurement uncertainty, $f$ is the computed velocity (Eq. (3)) and $u\left(x_{i}\right)$, is the individual uncertainty $i$ driven by the parameter $x_{i}$. This equation can also be expressed as given by equation (5) [1]:

$$
U_{v}=\sqrt{\sum_{1}^{n} U_{i}^{2}}
$$

where $U_{i}$ is the individual contribution of a specific uncertainty $i$ on the velocity measurement uncertainty. In the following sections, individual uncertainties are identified, and their individual influence on the velocity measurement uncertainty $U_{i}$ is determined. $U_{v}$ can be computed afterwards.

\section{Identification and quantification of the sources of uncertainties}

\subsection{Calibration uncertainties}

During the calibration procedure, which consists in finding the calibration factor calib [pix/mm], three sources of uncertainties are identified:

A first source of uncertainty is linked to the precision on the measurement of the reference distance $d_{c}[\mathrm{~mm}]$. This uncertainty is called reference length uncertainty.

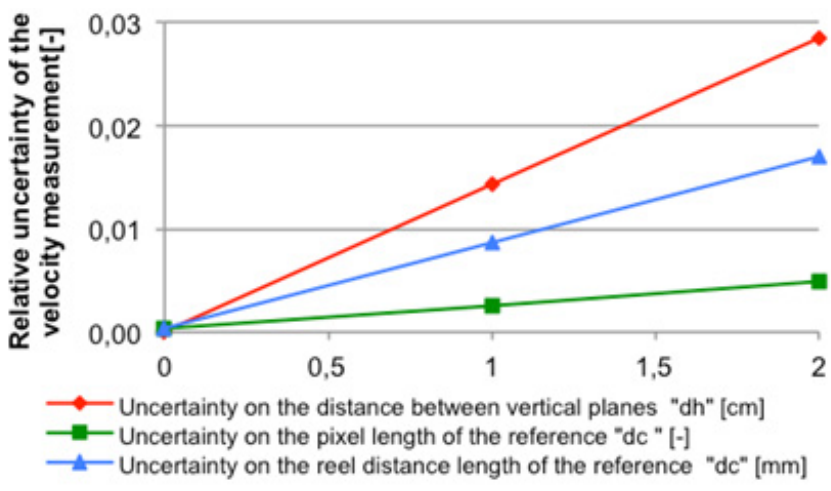

Fig. 3. Effect of the three calibration uncertainties on the relative uncertainty of the velocity measurement.

A second source of uncertainty is linked to the distance $d_{c}$ [pix]. Indeed, depending on the quality of the image, finding the exact pixels corresponding to the reference distance $d_{c}[\mathrm{~mm}]$ may only be possible up to a certain precision, linked to an uncertainty called reference pixel uncertainty.

Finally, a third source of uncertainty concerns the distance $d_{h}$ [mm]. Ideally, this value is null. Otherwise, an error is committed in the computation of calib $[\mathrm{pix} / \mathrm{mm}]$. The related uncertainty is called vertical plane uncertainty.

These three uncertainties can be quantified as a function of the error on $d_{c}[\mathrm{~mm}], d_{c}$ [pix] and $d_{h}[\mathrm{~mm}]$, respectively. The two first cases are solved by comparing the results of equation (3) with and without taking into account the uncertainty. Solving the third case requires to resolve the following Thales system:

$$
\frac{L}{L+d_{h} / 1000}=\frac{d_{c}}{d_{c, \text { corrected }}}
$$

where $d_{c \text {, corrected }}[\mathrm{mm}]$ is the corrected value of $d_{c}[\mathrm{~mm}]$ in the vertical projectile plane. The results are obtained by comparing the results of equation (3), using $d_{c}[\mathrm{~mm}]$ and $d_{\text {c, corrected }}[\mathrm{mm}]$. The results are presented on Figure 3. All the uncertainties evolve linearly as a function of the considered distances. The values of $d_{c}[\mathrm{~mm}], d_{c}$ [pix] and $d_{h}[\mathrm{~mm}]$ are estimated at $0.2 \mathrm{~mm}, 1$ pixel, and $20 \mathrm{~mm}$, respectively, for the presented experimental set-up. The induced relative uncertainties of the velocity measurement $U_{i}$ are equal to $0.11 \%, 0.26 \%$ and $0.29 \%$, respectively.

\subsection{Gamma angle uncertainty}

So far, the vertical projectile plane was always orthogonal to the axis of the camera. This section studies the likely case of a non-null angle $\gamma$, as schematised on Figure 4. The related uncertainty, called gamma angle uncertainty, consists in measuring the apparent distance $d_{p}[\mathrm{~mm}]$ instead of the real distance $y[\mathrm{~mm}]$. The resolution of this geometry leads to equation (7):

$$
y=\frac{d_{p}}{\cos \gamma+\tan \beta \sin \gamma} .
$$




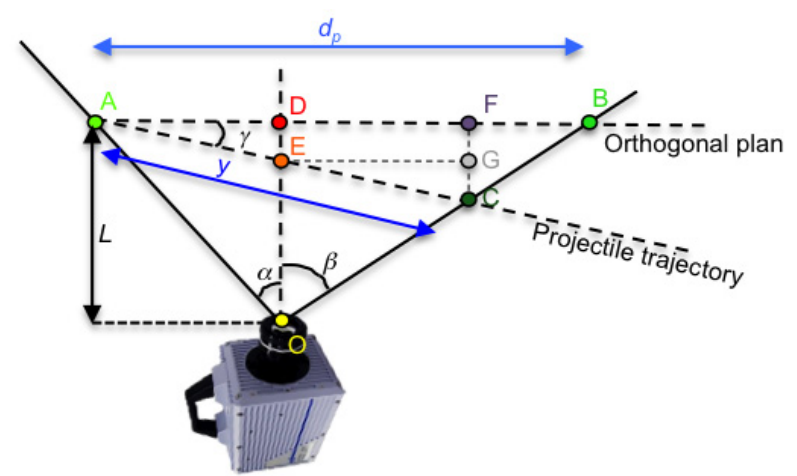

Fig. 4. Illustration of the geometry involved in the computation of the gamma angle uncertainty.

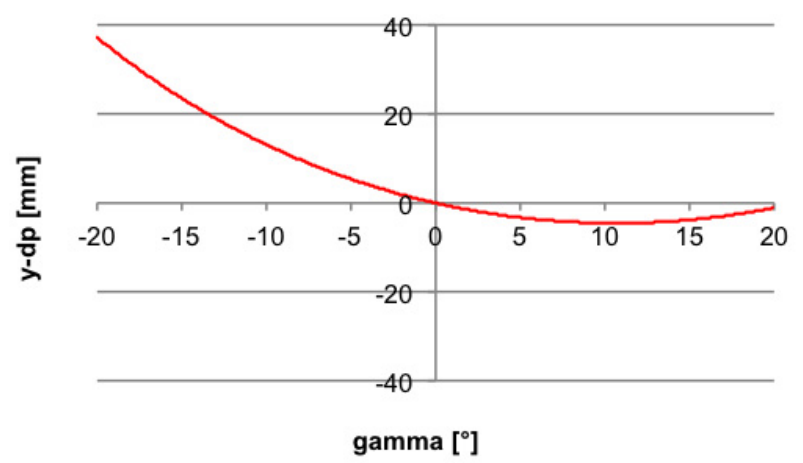

Fig. 5. Effect of the gamma angle uncertainty on the relative velocity measurement uncertainty of the velocity measurement.

In the present case, an extra assumption is made:

$$
[A D]=[B D]=\frac{d_{p}}{2}
$$

and thus,

$$
\beta=\tan ^{-1}\left(\frac{d_{p}}{2 L}\right) .
$$

The relation between the distance $d_{p}$ and $y$ is presented on Figure 5. This relation is not linear and not symmetrical around the vertical axis, due to the asymmetry of the geometry around the axis of the camera.

For the present set-up, the maximum uncertainty on the gamma angle is estimated at $3^{\circ}$.

Both the distances $d_{p}[\mathrm{~mm}]$ and $d_{c}[\mathrm{~mm}]$ are influenced by the gamma angle. The biggest error is committed when $d_{p}[\mathrm{~mm}]$ is measured with a gamma equals to $3^{\circ}$ and when $d_{c}[\mathrm{~mm}]$ is measured with a gamma equals to $-3^{\circ}$. Finally, the induced uncertainty is equals to $1.03 \%$.

\subsection{Uncertainty related to the measurement of $d_{p}$ [pix]}

An error can be committed while determining $d_{p}$ [pix] by choosing the pixels corresponding to the position of the projectile on the two images used for the velocity computation. As the projectile is moving, the motion blur $M B$

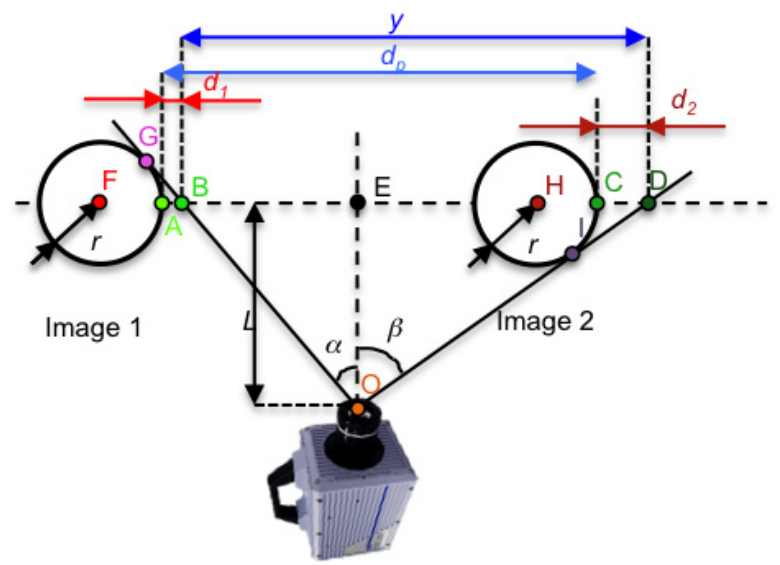

Fig. 6. Illustration of the geometry involved in the computation of the parallax error.

[pix] influences this error and the related uncertainty. Empirically, equation (10) has been determined for the presented set-up:

$$
U_{M B}=\frac{M B}{k_{M B}}=\frac{v_{p i x} S T}{k_{M B}}
$$

where $U_{M B}$ [pix] is the considered uncertainty, called motion blur uncertainty, and $k_{M B}[-]$ is an empirical coefficient that reduces the effect of the motion blur on the velocity measurement uncertainty. Among other things, its value depends on the quality of the image, the camera focus adjustment, the lighting and the contrast between the projectile and the background of the image. For the presented setup, its value has been empirically assessed at 2. This value has to be refined for each new experimental set-up. The relative uncertainty of the velocity measurement due to the motion blur is performed thanks to equation (3), computed with a nominal value $d_{p}$ [pix] and the corrected value $\left(d_{p}+U_{M B}\right)[\mathrm{pix}]$. The motion blur uncertainty is equal to $0.62 \%$.

\subsection{The parallax}

The parallax is the disparity between views of an object due to a difference in view points [6]. Consequently, when the camera is fixed, an object in motion like the considered projectile is viewed differently depending of its position. The concept of error instead of uncertainty is used here, as this error is fully defined by the geometry of the scene. The parallax is illustrated on Figure 6 and explained as follows. Proceeding to the measurement $d_{p}$ [pix], one has to locate a specific position of the projectile on two images, which induces two parallaxes. If the experimenter looks at the front of the projectile, he locates on image 1 the projection of the point $\mathrm{G}$ on the plane of the projectile's trajectory, which corresponds to the point B, instead of the point A. Similarly, he locates the point $\mathrm{D}$ instead of the point $\mathrm{C}$ on image 2. The parallax error consists in measuring $d_{p}$ [pix] and thus $d_{p}[\mathrm{~mm}]$ instead of $y=d_{p}-d_{1}+d_{2}[\mathrm{~mm}]$. 
As presented on Figure 6 , the distances $d_{1}[\mathrm{~mm}]$ and $d_{2}[\mathrm{~mm}]$ partially compensated each other. Therefore, the worst-case scenario occurs when one of these two distances is equal to zero, and the other is at its maximal value, which corresponds to the situation where $\alpha=0$ and $\beta=$ $\frac{\Omega}{2}$. The measurement $d_{p}[\mathrm{pix}]$ is then performed only on half of the image.

The result is:

$$
\begin{aligned}
\tan \beta & =\frac{d_{p}+d_{2}}{L} \\
\sin (90-\beta) & =\left(\frac{r}{r+d_{2}}\right) .
\end{aligned}
$$

For the present example, with a spherical projectile of a radius $r=40 \mathrm{~mm}$, the parallax error influence on the velocity measurement remains under $0.0002 \%$. An uncertainty on the radius $r$ leads to an even smaller uncertainty on the velocity and is therefore not considered here. In conclusion, to ensure a good measurement, one has to verify that the parallax remains low enough in comparison with the other uncertainties. Otherwise, it has to be taken into account.

\subsection{The perspective}

The perspective is used by a $\{$ camera-lens $\}$ system to represent three-dimensional objects and depth relationships on a two-dimensional surface. This representation goes with optical distortions that bring perspective errors. The quantification and correction of these errors is the topic of this section.

Mathematically, the perspective can be modelled as a system of transformations that changes the 3D realunit coordinates describing the filmed situation into $2 \mathrm{D}$ pixels coordinates describing the image. These transformations vary from one $\{$ camera-lens $\}$ system to another, and can be determined thanks to a geometrical calibration procedure, that quantifies the parameters of the chosen model. A well-known model in the literature is the pinhole model [7-11]. Its parameters can be classified in two types: the internal camera geometric and optical characteristics (intrinsic parameters) and the $3-\mathrm{D}$ position and orientation of the camera frame relative to a certain coordinate system (extrinsic parameters).

For the presented set-up, due to the orthogonality of the projectile plane with the axis of the camera, the extrinsic parameters are fully characterized by the parameters $L, \Omega$, and $R e s_{g}$, leading to a single calibration factor calib. The intrinsic parameters characterise the remaining distortions produced by the \{camera-lens $\}$ system (Fig. 7).

For example, a camera objective with a wide angle brings more distortions and induces more measurement errors than one with a normal angle of view. The intrinsic parameters of the $\{$ camera-lens $\}$ system can be determined by performing a calibration procedure based on a pin-hole model $[7,12]$. These parameters are then used to correct the distortion on the images generated by the camera. The difference between the measurements with

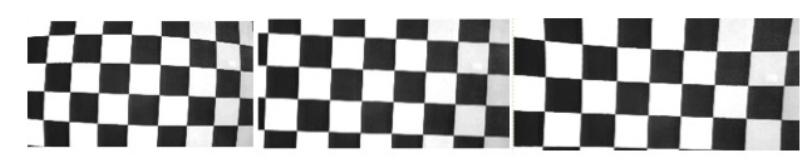

(a)

(b)

(c)

Fig. 7. Effect of lens distortion on an image. (a) Distorted image, (b) ideally projected image, (c) overly corrected image.

and without the correction of the distortion is considered as a fully characterised error called perspective error.

The calibration process is performed using an open source calibration toolbox for Matlab $[7,12]$. The principle is to take pictures of a calibration pattern and compare the dimensions on the image to the corresponding real dimensions. The parameters of the model are optimised to fit the image dimensions with the real dimensions. The employed distortion model is described on equation $(12)[7,12]$.

$$
\begin{aligned}
r_{d}^{2} & =x_{d}^{2}+y_{d}^{2} \\
r_{c o r} & =1+k_{1} r_{d}^{2}+k_{2} r_{d}^{4} \\
x_{c o r} & =\frac{f_{c x} x_{d}}{r_{c o r}}+p_{0 x} \\
y_{\text {cor }} & =\frac{f_{c y} y_{d}}{r_{c o r}}+p_{0 y}
\end{aligned}
$$

where

$-x_{d}$ [pix] and $y_{d}$ [pix] are the $x$-coordinate and $y$ coordinate of a considered point in the distorded image; $r_{d}[\mathrm{pix}]$ is the distance between this point and the centre of the image;

$-x_{\text {cor }}[\mathrm{pix}]$ and $y_{\text {cor }}[\mathrm{pix}]$ are the corrected $x$-coordinate and $y$-coordinate of the considered point; $r_{c o r}[\mathrm{pix}]$ is the distance between this point and the centre of the image;

- $f_{c x}$ [pix] and $f_{c y}[\mathrm{pix}]$ are the $x$-coordinate focal length and $y$-coordinate focal length of the camera [7,12];

$-p_{0 x}$ [pix] and $p_{0 y}$ [pix] are the $x$-coordinate principal point and $y$-coordinate principal point of the camera $[7,12]$;

$-k_{1} \quad$ [pix] and $k_{2}$ [pix] are the radial distortion parameters $[7,12]$.

More complex pin-hole models exist, which take into account the tangential distortions and higher degree radial distortions $[7,12]$. These distortions are not significant for the presented $\{$ camera-lens\} system.

The results obtained with the calibration toolbox are presented in Table 2. The induced distortion model is illustrated on Figure 8.

As one can see in Figure 8, the distortion is null at the centre of the image, and increases from the centre to the borders.

Besides, when $d_{c}$ [pix] and $d_{p}$ [pix] are measured in the same zone on the image (for example on the top left of the image), the distortions related to the two measurements are identical. Consequently, $d_{c}[\mathrm{pix}]$ and $d_{p}[\mathrm{pix}]$ are multiplied by the same distortion factor in equation (3). The induced error on the velocity due the distortion is 
Table 2. Values of the camera intrinsic parameters obtained thanks to the calibration procedure [12].

\begin{tabular}{ccc}
\hline Parameter & Value [pix] & Uncertainty $(3 \sigma)[\mathrm{pix}]$ \\
\hline$f_{c x}$ & 2685.88 & 10.24 \\
$f_{c y}$ & 2682.32 & 10.19 \\
$p_{0 x}$ & 526.26 & 5.68 \\
$p_{0 y}$ & 527.51 & 5.66 \\
$k_{1}$ & -0.13961 & 0.0241 \\
$k_{2}$ & -0.42466 & 0.4496 \\
\hline
\end{tabular}

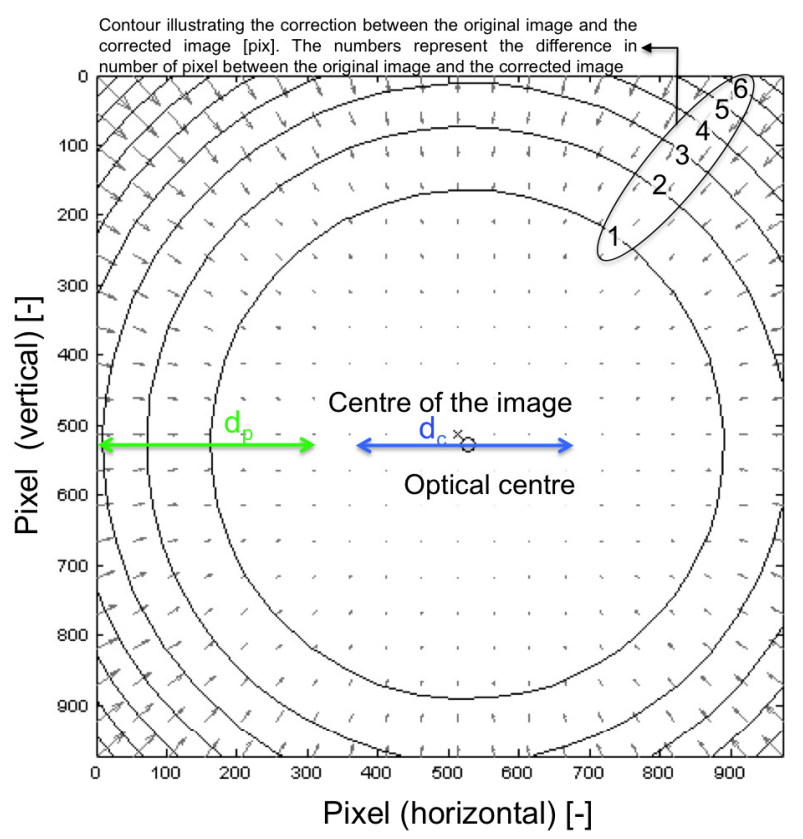

Fig. 8. Illustration of the distortion correction [12].

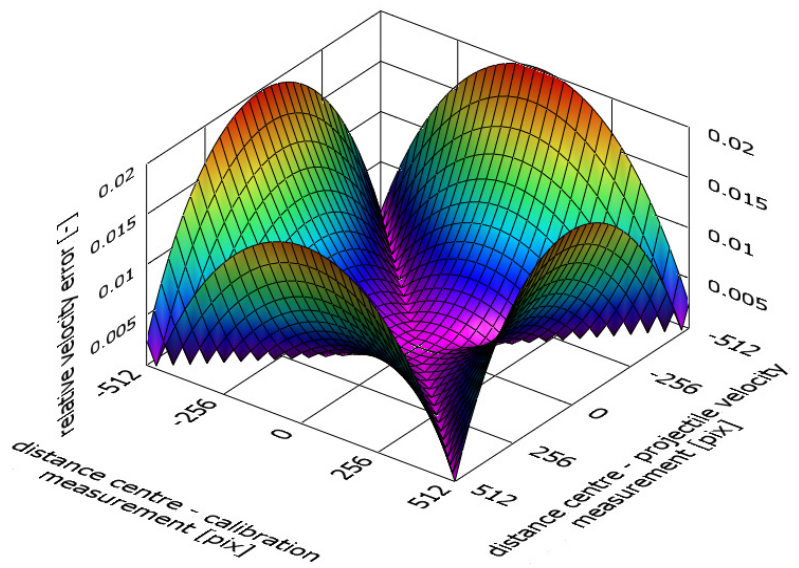

Fig. 9. Influence of the distances between the centre of the image and the measurements on the induced perspective error.

then null. If the distance between the two measurements increases, the error also increases.

All in all, the perspective error depends both on the location where the measurement on the image is performed and on the distance between $d_{c}$ and $d_{p}$ (Fig. 9).
For the present example, the quantification of the perspective error is assessed when $d_{c}$ [pix] is centred while $d_{p}$ [pix] is measured on the middle-left zone of the image (Fig. 8). The relative velocity error due to the perspective error is then equals to $0.77 \%$. Once the distortion is corrected, the only uncertainties that remain are based on the uncertainties on the parameters presented in Table 2. Supposing that all these uncertainties are independent, the relative distortion uncertainty $U_{\text {dist }}$ on the velocity measurement is computed using equation (13):

$$
U_{d i s t}=\sqrt{\sum_{1}^{n} U_{i, p e r s p}^{, 2}}
$$

where $U_{i, \text { persp }}$ are the relative uncertainties on the velocity measurement due to the uncertainties $i$ on the parameters presented in Table 2. The computed uncertainty is equal to $0.16 \%$.

The computed error and uncertainty value remain relatively low, due to the excellent quality of the camera. Nevertheless, they remain significant in comparison with the other identified uncertainties.

\subsection{Uncertainty on the chronometer of the camera}

This uncertainty called chrono uncertainty is linked to the precision of the chronometer $U_{c}$ [ns] of the camera. This value can be found in the datasheet of the camera. In the present case, this value is equal to $0.001 \%$ [3].

\subsection{Discretisation errors}

In every step of the velocity measurement process that involves a transformation of real distances into pixel distances, discretisation errors appear. Their effect is taken into account by rounding up the different computed uncertainties.

\section{Results and discussions}

All the results of the previous sections are gathered in Table 3.

Assuming that all these individual uncertainties are independent, the total relative uncertainty on the velocity measurement is obtained with equation (5), with and without correcting the perspective. These results lead to the following conclusions:

- For the proposed set-up, the most important source of uncertainty in terms of contribution to the total velocity uncertainty is the gamma uncertainty. Having a precise system to measure and reduce this angle is therefore essential.

- The second most important parameter is the perspective error. Even with an excellent camera, this error remains significant. The correction of the distortion is essential to reach a certain level of precision. 
Table 3. Global results of the uncertainty study.

\begin{tabular}{|c|c|c|c|c|}
\hline \multicolumn{2}{|c|}{ Type of uncertainty/error } & $\begin{array}{c}\text { Parameter that } \\
\text { drives this } \\
\text { uncertainty/error }\end{array}$ & $\begin{array}{c}\text { Value } \\
\text { of the } \\
\text { parameter }\end{array}$ & $\begin{array}{c}\text { Value of the induced } \\
\text { relative velocity } \\
\text { uncertainty/error }\end{array}$ \\
\hline \multicolumn{2}{|c|}{ Gamma angle } & $\gamma\left[{ }^{\circ}\right]$ (Fig. 4) & 3 & $1.01 \%$ \\
\hline \multirow{2}{*}{ Perspective } & $\begin{array}{c}\text { error (before } \\
\text { correction) }\end{array}$ & $\begin{array}{c}\text { [AC] [pix] } \\
\text { (Fig. 1) }\end{array}$ & $(-512,0)$ & $0.77 \%$ \\
\hline & $\begin{array}{c}\text { uncertainty } \\
\text { (after correction) }\end{array}$ & $U_{i, p e r s p}$ & Table 2 & $0.16 \%$ \\
\hline \multicolumn{2}{|c|}{ Motion blur } & $k_{M B}[-]$ & 2 & $0.62 \%$ \\
\hline \multicolumn{2}{|c|}{ Vertical plane } & $d_{h}[\mathrm{~mm}]$ & 20 & $0.29 \%$ \\
\hline \multicolumn{2}{|c|}{ Reference pixel } & $d_{c}[\mathrm{pix}]$ & 1 & $0.26 \%$ \\
\hline \multicolumn{2}{|c|}{ Reference length } & $d_{c}[\mathrm{~mm}]$ & 0.2 & $0.11 \%$ \\
\hline \multicolumn{2}{|c|}{ Chronometer } & $U_{c}[\mathrm{~ns}]$ & 10 & $0.001 \%$ \\
\hline \multicolumn{2}{|c|}{ Parallax } & $\begin{array}{l}\text { Radius } r \text { [mm] } \\
\text { (Fig. 5) }\end{array}$ & 40 & $0.0002 \%$ \\
\hline \multicolumn{4}{|c|}{ TOTAL (with perspective correction) } & $1.26 \%$ \\
\hline \multicolumn{4}{|c|}{ TOTAL (without perspective correction) } & $1.47 \%$ \\
\hline
\end{tabular}

Even with the correction, the perspective uncertainty remains significant.

- The motion blur uncertainty comes in the third place. Besides the empirical $k_{M B}[-]$ parameter, this uncertainty is directly influenced by the ST and the velocity of the projectile (Eq. (10)). The high-speed camera used in the proposed set-up is good enough to keep this value at an acceptable level.

- The three calibration uncertainties (vertical plane, reference pixel and reference length uncertainties) follow. In particular, the value of the vertical plane uncertainty can be reduced by designing a system that allow measuring precisely and reducing $d_{h}$.

- The parallax and the chrono uncertainties are insignificant for the proposed set-up.

Another experimental set-up, with other camera parameters, and different values of the parameters driving the individual uncertainties can lead to different conclusions. Therefore, for every set-up, all the presented mathematical tools should be used to identify the critical uncertainties and to improve the precision of the measurements. Integrating these computations in the tracking software allows systematically quantifying the uncertainty related to any camera measurement [2].

\section{Sensibility of the input parameters}

The sensibility studies allow the experimenter to refine the setup parameters presented in Table 1 in order to minimize the velocity measurement uncertainty.

In this section, sensibility studies of two input parameters presented in Table 1 are performed. These parameters are the distance of the camera $L$ and the travelling distance of the projectile $d_{p}$. The sensibility studies of the other parameters have been previously presented [2]. The measurements of $d_{c}$ [pix] and $d_{p}$ [pix] are performed on

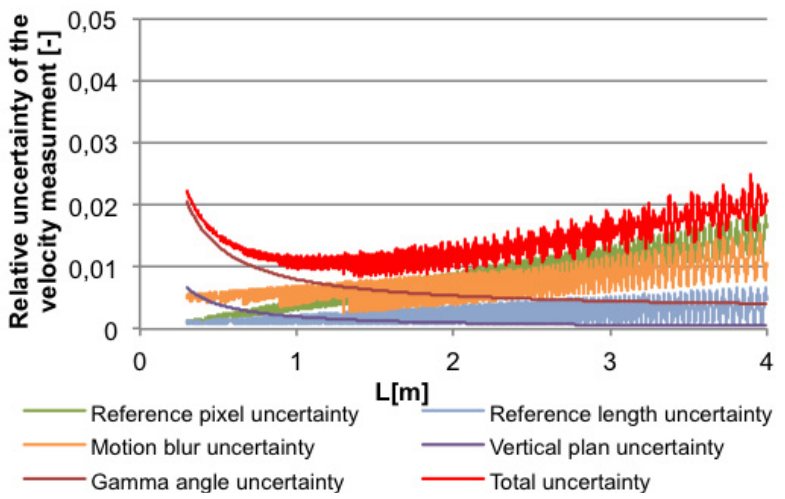

Fig. 10. Influence of the input parameter $L$ on the relative uncertainty of the velocity measurement.

the centre of the image in order to nullify the perspective and parallax errors.

\subsection{Sensibility of the $L$ parameter}

The results are presented on Figure 10. A low value of $L$ leads to a significant velocity measurement uncertainty, due to the individual contributions of the gamma angle uncertainty, and the vertical plane uncertainty. Besides, the contribution of the motion blur uncertainty, and the reference pixel uncertainty increases with $L$. As each pixel corresponds to a bigger real distance as $L$ increase, the measurement error of one pixel has more repercussion on the velocity measurement. For the same reason, oscillations on the curves characterizing pixels measurements (motion blur uncertainty, reference pixel uncertainty, and total uncertainty) also increase. These oscillations are due to discretisation errors, which increase as the distance represented by one pixel increases. 


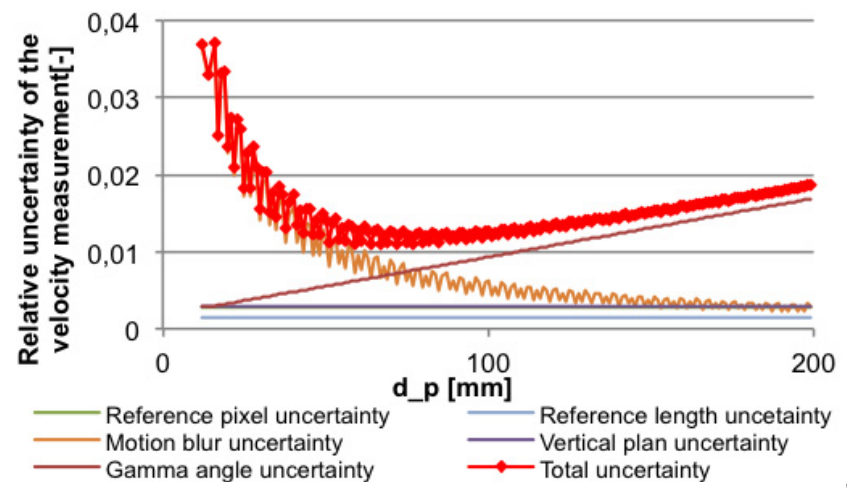

Fig. 11. Influence of the input parameter $d_{p}$ on the relative uncertainty of the velocity measurement.

The minimum velocity measurement uncertainty is observed for a distance $L$ included between $0.6 \mathrm{~m}$ and $1.3 \mathrm{~m}$, which corresponds to the value proposed in Table 1.

\subsection{Sensibility of the $d_{p}$ parameter}

The results are presented on Figure 11. When $d_{p}[\mathrm{~mm}]$ increases, $d_{p}$ [pix] also increases, and the impact of an error of one pixel due to the motion blur uncertainty is reduced. At the same time, the gamma angle uncertainty constantly increases.

When the distance $d_{p}$ becomes higher than $100 \mathrm{~mm}$, the contribution of gamma angle uncertainty angle continues to increase, and is not compensate by the reduction of the motion blur uncertainty, leading to an increase of the total uncertainty.

\section{Experimental validation}

To validate the computation, velocity measurements using the high-speed camera are compared with a reference measurement. Statistical indicators are compared with the computed uncertainty results. The set-up consists in measuring the velocity of a projectile flying at around $55 \mathrm{~m} / \mathrm{s}$. The velocity measurements are performed with the camera, as presented on Figure 1, and with an optical light screen barrier Drello LS9iN, frequently used in ballistic. A laser angle measurement system allow reducing the uncertainty on the gamma angle up to a value of $1^{\circ}$ instead of the aforementioned $3^{\circ}$. Eighteen projectiles are shot. The results are summarised on Figure 12. One can see the individual results in terms of relative difference between the light screen barrier measurements and the camera measurements. The camera velocity measurement uncertainty is estimated at $0.766 \%$, and the light screen barrier is characterized by an uncertainty on the velocity measurement of $1 \%[2,13]$. The total theoretical uncertainty of the relative difference between measurements, computed with equation (5), is equal to $1.26 \%$. The standard deviation of the relative difference between the measurements

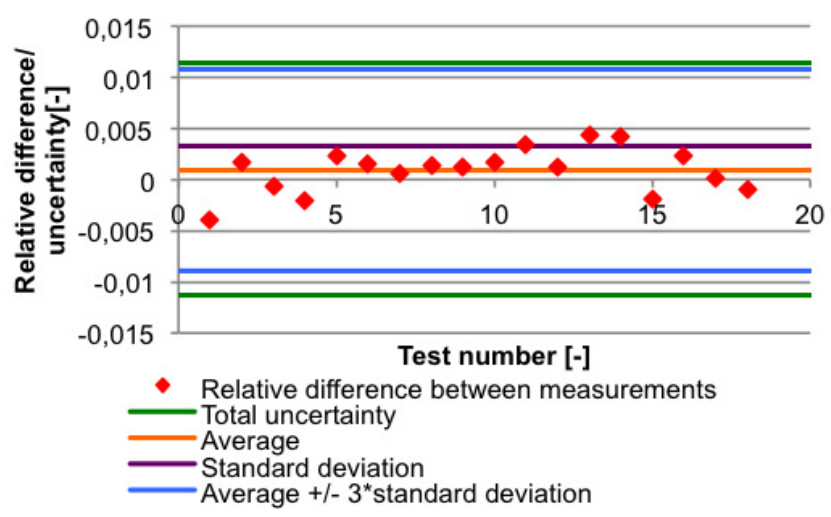

Fig. 12. Comparison of the uncertainty computed with the proposed approach and experimental measurements realised with a reference measurement.

is estimated thanks to the experimental samples and the statistical law $[2,14,15]$ :

$$
\sigma_{\text {diff }}^{2}=\sqrt{\frac{\sigma_{e c h}^{2}}{\chi^{2-1}(0,975 ; n-1)}}
$$

where $\sigma_{\text {diff }}$ is the standard deviation of the relative difference of measurements, $\sigma_{e c h}$ is the standard deviation of the presented sample, and $\chi^{2-1}$ is the $\mathrm{Khi}^{2}$ reverse law. The total theoretical uncertainty is compared with the confidence interval determined by the average plus or minus three times the standard deviation computed by equation (14). The theoretical uncertainty is very close to the confidence interval characterizing the measurements. The uncertainty computation approach is therefore reliable.

\section{Applying this study to continuous displacement}

All these uncertainty computation steps can be applied to the continuous measurement of the displacement of the projectile as a function of time (Fig. 13). This example shows the displacement measurement of a non-lethal deformable projectile impacting a rigid target.

The measurement is performed with the same experimental set-up, and is post-processed by the tracking software $[2,16]$. The obtained curve rises up to a maximum value, and then declines, characterizing the rebound of the projectile. The perspective and the parallax have not been considered. The uncertainty computations allow defining a corridor around the nominal curve in which the real measurement should figure. For relatively low displacements, the critical uncertainty is the motion blur uncertainty. For the maximal displacement, the critical uncertainty is the gamma uncertainty. These results are in perfect agreement with Figure 11. In conclusion, the precision of the results is fully characterised and is globally acceptable for the presented application. 

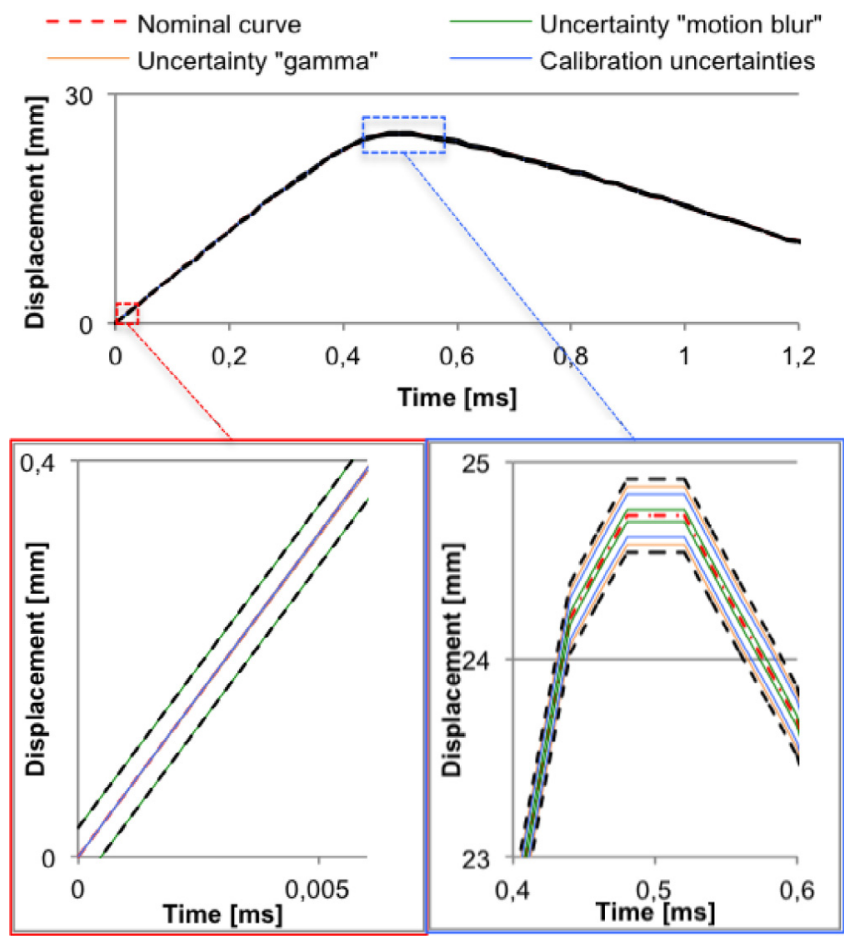

Fig. 13. Application of the uncertainty study on the continuous displacement of the projectile as a function of time measurement.

\section{Conclusions}

A complete theoretical uncertainty study on the velocity measurement of a flying projectile, using a high-speed camera, has been presented. All these results are applicable to other measurements performed by a camera. Many sources of uncertainties have been identified and quantified. On the one hand, the velocity measurement uncertainties have been quantified. On the other hand, it shows which aspects of the set-up should be improved to reduce the uncertainty. In particular, the authors draw the attention on the importance of mastering the geometry of the set-up, and on systematically correcting the distortions induced by the "camera-lens" system.

A sensibility study of two input parameters has been exposed, allowing the experimenter to optimize these parameters as to reduce the total velocity measurement uncertainty.

The results have been validated experimentally thanks to a reference velocity measurement. Good correspondence is observed.

Finally, all these results have been used to generate a confidence corridor around a continuous displacement measurement, as a function of time, using the high-speed camera. The results show how precise such measurements can be.

Thanks to the exposed study, the velocity measurements can achieve a very high degree of precision, and the uncertainty related to the performed measurement can be systematically quantified, which makes these measurements very robust and reliable. The methods can also help the buyer to dimension the requested material to obtain a desired uncertainty on the measurements.

\section{References}

1. Working group 1 of the Joint Committee for Guides in Metrology, JCGM100: 2008 évaluation des données de mesure - Guide pour l'expression de l'incertitude de mesure (2008)

2. C. Robbe, Évaluation experimentale de l'impact thoracique des projectiles non-létaux - Experimental Evaluation of the thoracic impact of non-lethal projectiles (Royal Military Academy (RMA), Université de Liège (ULg), 2013)

3. Photron, FAS TCAM S A 5 Specifications?: Partial Frame Rate/Recording Duration Table (2013), http:// www . photron.com/datasheet/FASTCAM_SA5.pdf

4. S. IVP, Machine Vision Introduction 206AD (2013), www. sick. com

5. C. Robbe, N. Nsiampa, A. Papy, A. Oukara, Practical considerations for using high-speed camera to measure dynamic deformation occurring at the impact of a kinetic energy non-lethal weapon projectile on ballistic simulant, in PASS 2012: Behind Armour Blunt Trauma

6. V. Nalwa, Outwardly Pointing Cameras, Tech Report from FullView.inc., Nuremberg, Germany, 2012, http://www . fullview.com/Outwardly_Pointing_Cameras.pdf (2013)

7. J. Bouguet, Visual methods for Three-dimensional modeling (California Institute of Technology, 1999)

8. O. Faugeras, Three-Dimensional Computer vision (MIT Press, Cambridge, 1993)

9. R. Hartley, A. Zisserman, Multiple View Geometry in Computer Vision (Cambridge Univsersity Press, 2004)

10. Y. Morvan, Acquisition, Compression and Rendering of Depth and Texture for Multi-View Video (Eindhoven University of Technology, 2009)

11. G. Bebis, Computer Vision (2013), http://www.cse.unr. edu/ ${ }^{\text {bebis / }}$

12. J. Bouguet, Camera Calibration Toolbox for Matlab, California Institute of Technology (2013), http://www . vision.caltech.edu/bouguetj/calib_doc/

13. M. Maldague, Contrôle des bases de vitesses de $0,25 \mathrm{~m}$, Internal Report (available by the authors, 2012)

14. E.D. Feigelson, G.J. Babu, in Modern Statistical Methods for Astronomy: With R Applications (Cambridge University Press, 2012), p. 490

15. W.H. Press, S.A. Teukolsky, W.T. Vetterling, B.P. Flannery, in Numerical Recipes: The Art of Scientific Computing, 3rd edn. (Cambridge University Press, 2007), p. 1256

16. C. Robbe, N. Nsiampa, A. Papy, A. Oukara, Practical considerations for using high-speed camera to measure dynamic deformation occurring at the impact of a kinetic energy non-lethal weapon projectile on ballistic simulant, in Personal Armour System Symposium (PASS) Conference Proceedings, Nuremberg, Germany, 2012

17. J. Tellinghuisen, Statistical error propagation, J. Phys. Chem. A 105, 3917-3921 (2001)

18. Solamen, Incertitude Sensibilité, Descriptif des pratiques dans l'industrie - Préconisations pour l'analyse thermique bâtiment (2011) 